\title{
Generator Matrices of Known Sequences and Study of Their Product
}

\author{
Dharmin R. Patel ${ }^{1}$, Sneha S. Kadiya ${ }^{2}$, Dr. Pradeep J Jha ${ }^{3}$ \\ '(Research Scholar, Calorx Teachers' University, Ahmedabad, INDIA) \\ ${ }^{2}$ (Research Scholar, Calorx Teachers' University, Ahmedabad, INDIA) \\ ${ }^{3}$ (Professor of Mathematics \& Research Guide, Rai University, Ahmedabad, INDIA)
}

\begin{abstract}
The prime concept of this paper is to introduce further algebraic operations on some known sequences and hence derive some new sequences. It further regulates these sequence by introducing procedural routines like identifying $n^{\text {th }}$ term using recurrence relation, deriving its general form, introducing an operator matrix and its further exponential operations, and establishing Eigen values relation between them. During the work, we have observed that in some cases the traits of original sequences pursue in the extended work on generator matrices of the new sequences thus formed.

Keywords: Pell sequence, Fibonacci sequence, Jha sequence, Operator Matrix, and Eigen values.
\end{abstract}

\subsection{Pell Sequence}

\section{Introduction To Some Known Sequences}

Much is said about the Pell sequence which originates from the Fermat's problem of finding general solution to search for integer values $\mathrm{x}$ and $\mathrm{y}$. Which satisfies $\mathrm{X}^{2}-2 \mathrm{Y}^{2}= \pm 1$. The ratio of the integer values satisfying $\mathrm{X}$ and $\mathrm{Y}$; which is $\frac{X}{V}$ appears as follows in terms of a sequence converging to $\sqrt{2}$.

It appears as $\frac{1}{1}, \frac{a}{2}, \frac{7}{5}, \frac{17}{12}, \frac{41}{29}, \ldots \ldots \ldots \ldots . . . .$.

As the terms advances the ratio becomes a better and better rational approximation to $\sqrt{2}$.

From the rational sequence above, we identify the terms appearing in the denominator.

Which are $1,2,5,12, \ldots \ldots$

We call the sequence as Pell sequence.

The terms of the sequence advances in atypical pattern; we call its general format as the recurrence relation

Also to Pell with uniformly of the sequences we are going to discuss in this note, we introduces ' 0 ' as the first term and rewrite the sequence as follows.

$$
P_{n}=0,1,2,5,12,29, \ldots \ldots
$$

[For the future reference to the Pell sequence, we shall consider the sequence given by the above reference (3)].

The recurrence relation to the sequence (3) is $\mathbf{t}_{\mathbf{n}+2}=\mathbf{2} \mathbf{t}_{\mathbf{n}+1}+\mathbf{t}_{\mathbf{n}} \forall \mathrm{n} \in N$; with $\mathrm{t}_{1}=0$, and $\mathrm{t}_{2}=1$,

Again, pursuing the reference of (3), the general term of the sequence $\left(=t_{n}\right)$ can be written as follows

The general term of the Pell sequence $P_{n}=\frac{\sqrt{2}}{4}\left[(1+\sqrt{2})^{n-1}-(1-\sqrt{2})^{n-1}\right]$ for $n \in N$

The generator matrix of the sequence is denoted as $\mathbf{P}$ is found from recurrence relation as follows.

$$
\left(\begin{array}{l}
P_{a} \\
P_{2}
\end{array}\right)=\left(\begin{array}{ll}
2 & 1 \\
1 & 0
\end{array}\right)\left(\begin{array}{l}
P_{2} \\
P_{1}
\end{array}\right) ; \mathbf{P}=\left(\begin{array}{ll}
2 & 1 \\
1 & 0
\end{array}\right)
$$

Where $\mathrm{P}_{\mathrm{i}}, \forall$ natural $i_{\text {are }}$ the given terms of Pell sequence.

\subsection{Jha Sequence:}

There are different approaches to introduce "Jha Sequence" which plays dominating role in understanding and analysing the terms of some standard sequences.

One of the most appealing appearance of the terms of Jha Sequence is found as follows. If we consider a set of Pythagorean triplets that format right triangles of Fermat family than the

inradius (=r) taken in increasing order of all such Fermat triangle then we get 'jha Sequence'.

For the sides $\mathrm{a}, \mathrm{b}$, and $\mathrm{h}[\mathrm{a}=$ shorter and $\mathrm{h}=$ hypotenuse $]$, a Fermat right triangle has the condition that $|\mathrm{b}-\mathrm{a}|=1$. Also, to establish symmetry with trigonometrical results we allow creeping of $a=0, b=1$ and $h=1$.

\begin{tabular}{|c|c|c|c|c|c|c|}
\hline \multirow[t]{2}{*}{ Sr. No. } & \multicolumn{3}{|c|}{ Sides of the triangle } & \multirow{2}{*}{$\begin{array}{c}\text { Area }=\Delta \\
\text { (a.b) } / 2\end{array}$} & \multirow[t]{2}{*}{$\mathrm{s}=(\mathrm{a}+\mathrm{b}+\mathrm{c}) / 2$} & \multirow{2}{*}{ in-radius $=r$} \\
\hline & a & $\mathbf{b}$ & $\mathbf{h}$ & & & \\
\hline 1 & 0 & 1 & 1 & 0 & 1 & 0 \\
\hline 2 & 3 & 4 & 5 & 6 & 6 & 1 \\
\hline 3 & 20 & 21 & 29 & 210 & 35 & 6 \\
\hline 4 & 119 & 120 & 169 & 7140 & 204 & 35 \\
\hline
\end{tabular}

With the inclusion of this, the Fermat family of right triangles is as follows

The terms of Jha sequence are $\mathrm{J}_{\mathrm{n}}=0,1,6,35,204$,

This sequence follows the recurrence relation

$\mathbf{J}_{\mathbf{n}+2}=\mathbf{6} \mathbf{J}_{\mathbf{n}+1}-\mathbf{J}_{\mathbf{n}}, n \in N$; from which we derive the general term which is as follows

The general term of Jha Sequence $=J_{n}=\frac{\sqrt{2}}{g}\left[(3+2 \sqrt{2})^{n-1}-(3-2 \sqrt{2})^{n-1}\right]$ for $n \in$

The generator matrix of the sequence is denoted as $\mathbf{J}$ is found from recurrence relation and it is as follow 


$$
\left(\begin{array}{l}
j_{a} \\
j_{2}
\end{array}\right)=\left(\begin{array}{cc}
6 & -1 \\
1 & 0
\end{array}\right)\left(\begin{array}{l}
j_{2} \\
j_{1}
\end{array}\right) ; \mathbf{J}=\left(\begin{array}{cc}
\mathbf{6} & -\mathbf{1} \\
1 & 0
\end{array}\right)
$$

\subsection{Fibonacci Sequence:}

The Fibonacci sequence is a known one since centuries. It has been derived during the study of propagation of rabbits in a pair of rabbits capable fairly enough to reproduction for a long period. It is claimed that the terms of the sequence show natural order of growth. This sequence has kept pace with time and has contributed profound concepts of mathematics. The term "Fibonacci numbers" is used to describe the sequence of numbers generated in the pattern

$1,1,2,3,5,8,13,21,34,55,89,144 \ldots$, where each number in the sequence is given by the sum of the previous two terms

This pattern is given by $F_{1}=1, F_{2}=1$ and the recursive formula $F_{n}=F_{n-1}+F_{n-2}, n>2$. The general term of Fibonacci sequence denoted as $\mathrm{F}_{\mathrm{n}}$ and given by

$$
\mathbf{F}_{\mathbf{n}}=\frac{1}{\sqrt{5}}\left[\left(\frac{1+\sqrt{5}}{2}\right)^{\mathrm{n}-1}-\left(\frac{1-\sqrt{5}}{2}\right)^{\mathrm{n}-1}\right] \text { for } n \in N \text {. }
$$

The generator matrix, denoted as ' $F$ ', of Fibonacci sequence is $F=\left(\begin{array}{ll}1 & 1 \\ 1 & 0\end{array}\right)$ satisfying the continuation of terms of the sequence

$$
\left(\begin{array}{l}
F_{2} \\
F_{2}
\end{array}\right)=\left(\begin{array}{ll}
1 & 1 \\
1 & 0
\end{array}\right)\left(\begin{array}{l}
F_{2} \\
F_{1}
\end{array}\right)
$$

\section{Product Of Generator Matrices And Analysis Of Result}

In this section we consider generator matrices of different sequences introduced in the above section and find their product. We observe that corresponding terms of resultant matrix and its exponential terms also observes mathematical symmetry and hence follow a regular pattern. i.e. a numerical integral sequence. We again find the generator matrix to such obtained new sequence.

It will be interesting to note that Eigen values of all such matrices in increasing order of exponents also follow a regular pattern which also can be studied to pursue some mathematical pattern.

2.1 Product of Generator matrices of Pell and Jha sequence:

As said earlier, we consider from the above unit, the generator matrix $P=\left(\begin{array}{ll}\mathbf{2} & \mathbf{1} \\ \mathbf{1} & \mathbf{0}\end{array}\right)$ of Pell sequence and $\mathrm{J}=\left(\begin{array}{cc}\mathbf{6} & \mathbf{- 1} \\ \mathbf{1} & \mathbf{0}\end{array}\right)$ of Jha

sequence and find their product PJ as follows.

The product of $P$ and $J$ matrices denoted as $[\mathrm{PJ}]$

$[P J]=\left(\begin{array}{ll}2 & 1 \\ 1 & 0\end{array}\right)\left(\begin{array}{cc}6 & -1 \\ 1 & 0\end{array}\right)=\left(\begin{array}{cc}13 & -2 \\ 6 & -1\end{array}\right)$

We compute different powers of the matrix $\mathrm{PJ}$ and enlist the result as follow.

$$
\begin{aligned}
& {[\mathrm{PJ}]^{1}=\left(\begin{array}{cc}
13 & -2 \\
6 & -1
\end{array}\right) \quad[P J]^{2}=\left(\begin{array}{cc}
157 & -24 \\
72 & -11
\end{array}\right) \quad[P J]^{a}=\left(\begin{array}{cc}
1897 & -290 \\
870 & -133
\end{array}\right)} \\
& {[P J]^{4}=\left(\begin{array}{ll}
22921 & -3504 \\
10512 & -1607
\end{array}\right)}
\end{aligned}
$$

And hence in general we have a sequence of matrix as follows.

PJ: $[\mathrm{PJ}]^{1},[\mathrm{PJ}]^{2},[\mathrm{PJ}]^{3},[\mathrm{PJ}]^{4}$

Where different exponents are shown as above

Hence as it can be observed that for different exponents,

$\mathrm{t}_{1}=1$ from $\left.[\boldsymbol{P}]\right]$

$\mathrm{t}_{2}=11$ from $[\boldsymbol{P J}]^{2}$

$\mathrm{t}_{3}=133$ from $[\boldsymbol{P J}]^{\mathrm{a}}$

$\mathrm{t}_{4}=1607$ from $[\boldsymbol{P} \boldsymbol{J}]^{4}$

The recurrence relation is $t_{n+2}=\mathbf{1 2} t_{n+1}+t_{n}$ with $t_{1}=\mathbf{1}$ and $t_{2}=\mathbf{1 1}$

We observe that all the entries of the matrix $[\boldsymbol{P} \boldsymbol{J}]^{n}$ are expressed in terms of the sequence $\left(\mathrm{t}_{\mathrm{n}}\right)$

$$
[\boldsymbol{P J}]^{\mathrm{n}}=\left(\begin{array}{cc}
13 \sum_{i=1}^{n} t_{i}+\sum_{i=1}^{n-1} t_{\mathrm{i}} & -2 \sum_{i=1}^{n} t_{i} \\
\mathbf{6} \sum_{i=1}^{n} t_{i} & -t_{n}
\end{array}\right) \text { Where } \mathbf{t}_{\mathbf{1}}=\mathbf{1}, \mathbf{t}_{\mathbf{2}}=\mathbf{1 1} \text { for all } \mathbf{n} \geq \mathbf{2}
$$

Where $[\mathrm{PJ}]^{1}=\left(\begin{array}{cc}\mathbf{1 3} & -\mathbf{2} \\ \mathbf{6} & -\mathbf{1}\end{array}\right)$

The terms of the sequence $\left(=\mathrm{t}_{\mathrm{n}}\right)$ is expressed as follows.

$\left(t_{n}\right)=1,11,133,1607,19417 \ldots$

The sequence has two dominant features as follows.

(1) With $t_{1}=1, t_{2}=11$ and the recurrence relation is

$$
\mathbf{t}_{\mathrm{n}+2}=12 \mathbf{t}_{\mathrm{n}+1}+\mathbf{t}_{\mathrm{n}} \forall \mathrm{n} \in N
$$

Also its general term is

$$
t_{n}=\frac{1}{2 \sqrt{37}}\left\{(5+\sqrt{37})(6+\sqrt{37})^{n-1}-(5-\sqrt{37})(6-\sqrt{37})^{n-1}\right)
$$


In order to make further analysis we study the sequence given by the result (15) and name it sequence S1.

$\mathrm{S} 1:=1,11,133,1607,19417 \ldots$

We have its generator matrix $\left(\begin{array}{cc}\mathbf{1 2} & \mathbf{1} \\ \mathbf{1} & \mathbf{0}\end{array}\right)=\mathbf{V}$ say Let its terms be denoted as $\mathrm{t}_{\mathrm{i}}$ for all $\mathrm{i} \in \mathbb{N}$.

$\left(\begin{array}{l}t_{a} \\ t_{2}\end{array}\right)=\left(\begin{array}{cc}12 & 1 \\ 1 & 0\end{array}\right)\left(\begin{array}{c}t_{2} \\ t_{1}\end{array}\right)=V\left(\begin{array}{c}t_{2} \\ t_{1}\end{array}\right)$

$\left(\begin{array}{l}t_{4} \\ t_{a}\end{array}\right)=\left(\begin{array}{cc}12 & 1 \\ 1 & 0\end{array}\right)^{2}\left(\begin{array}{l}t_{2} \\ t_{1}\end{array}\right)=\left(\begin{array}{cc}145 & 12 \\ 12 & 1\end{array}\right)\left(\begin{array}{l}t_{2} \\ t_{1}\end{array}\right)$ and so $\quad V^{2}=\left(\begin{array}{cc}145 & 12 \\ 12 & 1\end{array}\right)$

$\left(\begin{array}{l}t_{5} \\ t_{4}\end{array}\right)=\left(\begin{array}{cc}12 & 1 \\ 1 & 0\end{array}\right)^{3}\left(\begin{array}{l}t_{2} \\ t_{1}\end{array}\right)=\left(\begin{array}{cc}1752 & 145 \\ 145 & 12\end{array}\right)\left(\begin{array}{l}t_{2} \\ t_{1}\end{array}\right)$ and $s o v^{3}=\left(\begin{array}{cc}1752 & 145 \\ 145 & 12\end{array}\right)$

Similarly $V^{4}=\left(\begin{array}{cc}\mathbf{1 2} & \mathbf{1} \\ \mathbf{1} & \mathbf{0}\end{array}\right)^{4}=\left(\begin{array}{cc}21169 & 1752 \\ 1752 & 145\end{array}\right)$

Continuing in the same pattern, we have

$$
\left(\begin{array}{c}
t_{n} \\
t_{n-1}
\end{array}\right)=\left(\begin{array}{cc}
12 & 1 \\
1 & 0
\end{array}\right)^{n-2}\left(\begin{array}{c}
t_{2} \\
t_{1}
\end{array}\right) \text { or }\left(\begin{array}{c}
t_{n+2} \\
t_{n+1}
\end{array}\right)=\left(\begin{array}{cc}
12 & 1 \\
1 & 0
\end{array}\right)^{n}\left(\begin{array}{c}
t_{2} \\
t_{1}
\end{array}\right) \text { for } n \in N
$$

We have a sequence of generator matrices as follows

$\left(\begin{array}{cc}12 & 1 \\ 1 & 0\end{array}\right),\left(\begin{array}{cc}145 & 12 \\ 12 & 1\end{array}\right),\left(\begin{array}{cc}1752 & 145 \\ 145 & 12\end{array}\right),\left(\begin{array}{cc}21169 & 1752 \\ 1752 & 145\end{array}\right), \ldots \ldots \ldots \ldots$

In order to write the general form of the matrix sequence, we take a sequence of its positional terms as follows.

$0,1,12,145,1752 \ldots \ldots \ldots \ldots$

$T_{1}=0, T_{2}=1$ with a recurrence relation $T_{n+2}=12 T_{n+1}+T_{n}$

(19)

This helps write the general term of the matrix sequence.

$\therefore[V]^{n}=\left(\begin{array}{cc}12 & 1 \\ 1 & 0\end{array}\right)^{\mathrm{n}}=\left(\begin{array}{cc}T_{n+2} & T_{n+1} \\ T_{n+1} & T_{n}\end{array}\right)$ for all $\mathrm{n} \in N$

$\therefore[V]^{n}=\left(\begin{array}{cc}12 & 1 \\ 1 & 0\end{array}\right)^{n}=\left(\begin{array}{cc}\sum_{i=1}^{n+1} t_{i} & \sum_{i=1}^{n} t_{i} \\ \sum_{i=1}^{n} t_{i} & \sum_{i=1}^{n-1} t_{i}\end{array}\right)_{2 \times 2}$ For $n \geq 2$ and $V=\left(\begin{array}{cc}12 & 1 \\ 1 & 0\end{array}\right)$

Now we enlist the result of Eigen values to the different exponents of the matrix $\mathrm{V}^{\mathrm{n}}$.

Matrix

Eigen values

$\mathrm{V} \quad(\mathrm{EV})^{1}=(\mathbf{6} \pm \mathbf{1} \sqrt{\mathbf{3 7}})$

$\mathrm{V}^{2} \quad(\mathrm{EV})^{2}=(\mathbf{7 3} \pm \mathbf{1 2} \sqrt{\mathbf{3 7}})$

$\mathrm{V}^{3}$

$(E V)^{3}=(882 \pm 145 \sqrt{37})$

$\mathrm{V}^{\mathrm{n}}$

$$
(E V)^{\mathrm{n}}=\left[\left(6 \sum_{i=1}^{\mathrm{n}} t_{\mathrm{i}}+\sum_{\mathrm{i}=1}^{\mathrm{n}} t_{\mathrm{i}}\right) \pm\left(\sum_{\mathrm{i}=1}^{\mathrm{n}} t_{\mathrm{i}}\right) \sqrt{37}\right]
$$

We make the following observations from the pattern of Eigen values.

(1) The real value $\sqrt{37}$ appears on the Eigen values of all the matrices. Considering a generalized form of the generator matrix $\mathrm{V}^{\mathrm{n}}$;

we put its Eigen value as $\boldsymbol{\alpha}_{\mathbf{n}}+\boldsymbol{\beta}_{\mathrm{n}} \sqrt{37}$

for different values of $\mathrm{n} \in \mathbb{N}$. 
Now, we note an important point that $\lim _{n \rightarrow \infty} \frac{\left(a_{n}\right)}{(\beta n)}$ is a rational approximation of $\sqrt{37}$ the approximating sequence is $\frac{6}{1^{x}}$

$\frac{72}{12}, \frac{892}{145}, \frac{10657}{1752}$

(2) From the two parts (rational and irrational) of Eigen values, we have two different pattern of sequences $6,73,882,10657 \ldots \ldots .$. and $1,12,145,1752$.

The important point is that the terms of these two sequences follow the same recurrence relation which is given in (13).

\subsection{Product of Generator matrices of Fibonacci sequence and Jha Sequence:}

As said earlier, we consider from the above unit, the generator matrix $F=\left(\begin{array}{ll}1 & 1 \\ 1 & 0\end{array}\right)$ of Fibonacci sequence and $\mathrm{J}=\left(\begin{array}{cc}\mathbf{6} & -\mathbf{1} \\ \mathbf{1} & \mathbf{0}\end{array}\right)$ of Pell sequence and find their product FJ as follows

The product of $\mathbf{J}$ and $\mathbf{F}$ matrices denoted as $[\mathrm{FJ}]$

$$
[F]=\left(\begin{array}{ll}
1 & 1 \\
1 & 0
\end{array}\right)\left(\begin{array}{cc}
6 & -1 \\
1 & 0
\end{array}\right)=\left(\begin{array}{cc}
7 & -1 \\
6 & -1
\end{array}\right)
$$

We compute different powers of the matrix PJ and enlist the result as follow.

$$
[F J]^{2}=\left(\begin{array}{ll}
43 & -6 \\
36 & -5
\end{array}\right) \quad[F J]^{a}=\left(\begin{array}{ll}
265 & -37 \\
222 & -31
\end{array}\right) \quad[F J]^{4}=\left(\begin{array}{cc}
1633 & -228 \\
1368 & -191
\end{array}\right)
$$

And hence in general we have a sequence of matrix as follows.

FJ: $[\mathbf{F J}]^{1},[\mathbf{F J}]^{2},[\mathbf{F J}]^{3},[\mathbf{F J}]^{4}$

Where different exponents are shown as above

Hence as it can be observed that for different exponents,

$\mathrm{t}_{1}=1$ from $\left.[F]\right]$

$\mathrm{t}_{2}=5$ from $\left.[F]\right]^{2}$

$\mathrm{t}_{3}=31$ from $\left.[F]\right]^{a}$

$\mathrm{t}_{4}=191$ from $\left.[\boldsymbol{F}]\right]^{4}$

The recurrence relation is $t_{n+2}=6 t_{n+1}+t_{n}$ with $t_{1}=1$ and $t_{2}=5$

We observe that all the entries of the matrix $[F J]^{n}$ are expressed in terms of the sequence $\left(t_{n}\right)$

$[F J]^{n}=\left(\begin{array}{cc}7 \sum_{i=1}^{n} t_{i}+\sum_{i=1}^{n-1} t_{i} & -\sum_{i=1}^{n} t_{i} \\ 6 \sum_{i=1}^{n} t_{i} & -t_{n}\end{array}\right) \quad$ Where $t_{1}=1, \mathbf{t}_{2}=\mathbf{5}$ for all $\mathbf{n} \geq \mathbf{2}$

Where $[\mathrm{FJ}]^{1}=\left(\begin{array}{ll}\mathbf{7} & -1 \\ 6 & -1\end{array}\right)$

The sequence $t_{n}$ is expressed as follows.

$\left(t_{n}\right)=1,5,31,191,1177$.

For which two dominant features are as follows.

(1) With $t_{1}=1, t_{2}=5$ and the recurrence relation is

$\mathbf{t}_{\mathrm{n}+2}=\mathbf{6} \mathbf{t}_{\mathrm{n}+1}+\mathbf{t}_{\mathrm{n}} \forall \mathrm{n} \in \mathbb{N}$

Also its general term is

$t_{n}=\frac{1}{2 \sqrt{10}}\left\{(2+\sqrt{10})(3+\sqrt{10})^{n-1}-(2-\sqrt{10})(3-\sqrt{10})^{n-1}\right\}$

In order to make further analysis we study the sequence given by the result calling it a sequence $\mathrm{S} 1$,

We have its generator matrix $\left(\begin{array}{ll}6 & 1 \\ 1 & 0\end{array}\right)=\mathbf{Y}$ say Let its terms be denoted as $t_{i}$ for all $i \in N_{x}$

$\left(\begin{array}{l}t_{2} \\ t_{2}\end{array}\right)=\left(\begin{array}{ll}6 & 1 \\ 1 & 0\end{array}\right)\left(\begin{array}{l}t_{2} \\ t_{1}\end{array}\right)=Y\left(\begin{array}{l}t_{2} \\ t_{1}\end{array}\right)$

$\left(\begin{array}{l}t_{4} \\ t_{2}\end{array}\right)=\left(\begin{array}{ll}6 & 1 \\ 1 & 0\end{array}\right)^{2}\left(\begin{array}{l}t_{2} \\ t_{1}\end{array}\right)=\left(\begin{array}{cc}37 & 6 \\ 6 & 1\end{array}\right)\left(\begin{array}{l}t_{2} \\ t_{1}\end{array}\right)$ and so, $Y^{2}=\left(\begin{array}{cc}37 & 6 \\ 6 & 1\end{array}\right)$

Similarly,

$\left(\begin{array}{l}t_{5} \\ t_{4}\end{array}\right)=\left(\begin{array}{ll}6 & 1 \\ 1 & 0\end{array}\right)^{3}\left(\begin{array}{l}t_{2} \\ t_{1}\end{array}\right)=\left(\begin{array}{cc}228 & 37 \\ 37 & 6\end{array}\right)\left(\begin{array}{l}t_{2} \\ t_{1}\end{array}\right)$ and so, $Y^{3}=\left(\begin{array}{cc}228 & 37 \\ 37 & 6\end{array}\right)$

Continuing in the same pattern, we have

$$
\left(\begin{array}{c}
t_{n} \\
t_{n-1}
\end{array}\right)=\left(\begin{array}{ll}
6 & 1 \\
1 & 0
\end{array}\right)^{n-2}\left(\begin{array}{l}
t_{2} \\
t_{1}
\end{array}\right) \text { or }\left(\begin{array}{l}
t_{n+2} \\
t_{n+1}
\end{array}\right)=\left(\begin{array}{ll}
6 & 1 \\
1 & 0
\end{array}\right)^{n}\left(\begin{array}{l}
t_{2} \\
t_{1}
\end{array}\right) \text { for } n \in \mathbb{N}
$$

We have sequence of generator matrices as follows 
In order to write the general form of the matrix sequence, we take a sequence of its positional terms as follows.

$0,1,6,37,228,1331$

$T_{1}=0, T_{2}=1$ with a recurrence relation $T_{n+2}=6 T_{n+1}+T_{n}$

$$
\therefore Y]^{n}=\left(\begin{array}{ll}
6 & 1 \\
1 & 0
\end{array}\right)^{n}=\left(\begin{array}{cc}
\sum_{i=1}^{n+1} t_{i} & \sum_{i=1}^{n} t_{i} \\
\sum_{i=1}^{n} t_{i} & \sum_{i=0}^{n-1} t_{i}
\end{array}\right)_{2 \times 2} \quad \text { For } n \geq 2 \text { and } Y=\left(\begin{array}{ll}
6 & 1 \\
1 & 0
\end{array}\right)
$$

Now we enlist the result of Eigen values to the different exponents of the matrix $\mathrm{Y}^{\mathrm{n}}$.

$$
\begin{array}{ll}
\text { Matrix } & \begin{array}{c}
\text { Eigen values } \\
\mathrm{Y}
\end{array} \\
\mathrm{Y}^{2} & (\mathrm{EY})^{1}=(3 \pm 1 \sqrt{\mathbf{1 0}}) \\
\mathrm{Y}^{3} & (\mathrm{EY})^{3}=(117 \pm \mathbf{3 7} \sqrt{\mathbf{1 0}}) \\
\mathrm{Y}^{\mathrm{n}} & \\
\mathrm{Y}^{\mathrm{n}} & \left.(\mathrm{EY})^{\mathrm{n}}=\left[\left(3 \sum_{\mathrm{i}=1}^{n} t_{\mathrm{i}}\right)+\sum_{\mathrm{i}=0}^{n-1} t_{\mathrm{i}}\right) \pm\left(\sum_{\mathrm{i}=1}^{n} t_{\mathrm{i}}\right) \sqrt{10}\right] \text { for } n \in \mathbb{N}
\end{array}
$$

We make the following observation from the pattern of Eigen values.

(3) The real value $\sqrt{10}$ remains constant and appears on the Eigen values of all the matrices. Considering a generalized form of the generator matrix $\mathrm{Y}^{\mathrm{n}}$; we put its Eigen

value as $\alpha_{n}+\beta_{n} \sqrt{10}$ for different values of $n \in \mathbb{N}$

Now, we note an important point that $\lim _{n \rightarrow \infty} \frac{\left(\alpha_{n}\right)}{(0 n)}$ is a rational approximation of $\sqrt{10}$ the approximating sequence is $\frac{1}{1} \frac{19}{6}, \frac{117}{97}, \frac{721}{228}{ }^{x} \ldots \ldots$

(4) From the two parts (ration and irrational) of Eigen values, we have two different pattern of sequence $3,19,117,721 \ldots \ldots$. and 1 , $6,37,228 \ldots \ldots \ldots . . .$.

The important point is that the terms of these two sequences follow the same recurrence relation which is given in (24).

\section{Conclusion}

The content of different units of this paper lays down a new road on which many new sequences having traits of parent sequences can be generated. The important role in the pursuance of the logic is the one about generator matrices of the original sequences. As it is known that the matrix product is non-commutative in algebraic nature, we get enough scope to find more sequences and their generator matrices with their properties like that of Eigen values and Eigen vectors. This leaves the topic open - ended for the other known sequences also.

Vision: Our next topic relates the same notion but with different known sequences.

\section{References}

[1]. T.Koshy, Fibonacci and Lucas Numbers with Applications (John Wiley, New York, 2001)

[2]. A. F. Horadam, Pell identities, Fibonacci Quart.,9(3) (1971),245-263.

[3]. V.A. Achesariya, Dr. P.J. Jha, "Set of Pythagorean Triplets", IOSR Journal of Mathematics (IOSR-JM); p-ISSN: 2319-7665X. vol-11,pp51-60.

[4]. Kadiya S.S, Patel D.R. , Dr. P. J. Jha, “ Matrix Operation on generator matrices of known sequences and important derivations" International Journal of Applied Research 2016:2(7);p-ISSN: 2394-7500:pp933-938.

[5]. Patel D.R., Kadiya S.S., Patel C.K., Dr.P.J.Jha; "Member Triangles of Fermat Family and Geometric Inter-relationship with Standard Sequences" ISBN.978-2-642-24819-9

[6]. Proceedings of the International Conference on Emerging Trends in Scientific Research (ICETR-2015) 